\title{
Propionate production from fermentation of selected $\beta$-glucans by gut microbiota in vitro
}

\author{
H.C. Harris ${ }^{1}$, C.A. Edwards ${ }^{1}$ and D.J. Morrison ${ }^{2}$ \\ ${ }^{1}$ School of Medicine, College of Medical, Veterinary and Life Sciences, University of Glasgow, New Lister Building, \\ Alexander Parade Glasgow, UK, G31 2ER and ${ }^{2}$ SUERC, University of Glasgow, East Kilbride, UK, G75 OQF
}

The short chain fatty acids (SCFA); acetate, propionate, and butyrate are produced by colonic fermentation of non-digestible carbohydrates (NDC) by the gut microbiota. Propionate is thought to play a prominent role in metabolic health and has recently been shown to contribute to intestinal gluconeogenesis ${ }^{(1)}$, and appetite regulation ${ }^{(2)}$. These effects are thought to be mediated, at least in part, by SCFA induced activation of free fatty acid receptors 2 and 3 (FFAR2/3). Identifying NDC that increase the production of propionate may be advantageous to metabolic health ${ }^{()}$. Beta $(\beta)$ glucans are NDC polysaccharides composed solely of glucose and have been linked with increased propionate production in vitro ${ }^{(3,4)}$. This study aimed to establish the extent of propionate production from a range of different sources of $\beta$-glucan from oats, barley and algae with varied structures and bond.

Batch in vitro fermentations ( $\mathrm{n}=3 \mathrm{M} / \mathrm{F} 2 / 1,23-26$ years) were conducted with a selection of commercially available $\beta$-glucans; Oats ( $\beta 1-3,1-4)$ : Oatwell 22, Oatwell 28, Promoat, Barley $(\beta 1-3,1-4)$ : Glucagel and Algae: Laminarin $(\beta 1-3,1-6)$ with inulin as a control. SCFA were extracted in ether and measured by GC-FID with 2-ethylbutyric acid as internal standard. Statistical analysis was conducted using one way ANOVA with post hoc Bonferroni.

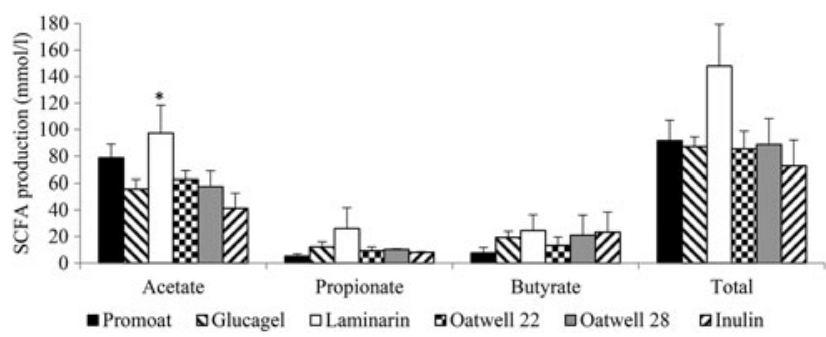

Fig. 1. SCFA production from $\beta$-glucan fermentation. (mean \pm SEM). * indicates $p=0.052$ vs inulin.

All $\beta$-glucans analysed were well fermented yielding similar total SCFA as inulin. The source of $\beta$-glucan had no significant effect on SCFA production (Figure 1) or the molar proportion of propionate. More detailed analysis is needed before these NDCs could be considered to be 'propiogenic' for use as a functional food in metabolic health.

This work was supported by BBSRC DRINC.

1. De Vadder F, Kovatcheva-Datchary P, Goncalves D, et al., (2014) Cell 156: 84-96.

2. Chambers E, Viardot A, Psichas A, et al. (2014) Gut 64: 1-11.

3. Deville C, Gharbi M, Dandrifosse G, et al. (2007) J Sci Food Agric, 1725: 1717-1725.

4. Queenan KM, Stewart M, Smith K, et al. (2007) Nutr J 6:6 